\title{
Polarization-Entangled Photon Pairs Using Spontaneous Four-Wave Mixing in a Fiber Loop
}

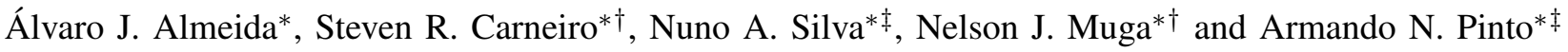 \\ *Instituto de Telecomunicações, Campus Universitário de Santiago, 3810-193, Aveiro, Portugal \\ ${ }^{\dagger}$ Department of Physics, University of Aveiro, Campus Universitário de Santiago, 3810-193, Aveiro, Portugal \\ ${ }^{\ddagger}$ Department of Electronics, Telecommunications and Informatics, University of Aveiro, \\ Campus Universitário de Santiago, 3810-193, Aveiro, Portugal \\ Emails: aalmeida@av.it.pt,.src@av.it.pt, nasilva@av.it.pt, muga@av.it.pt, anp@ua.pt
}

\begin{abstract}
We generate polarization-entangled photon pairs in the 1550-nm wavelength telecom band, using spontaneous fourwave mixing in a highly nonlinear fiber loop. With accidental coincidences subtracted, we obtain coincidence fringes with visibilities greater than $86 \%$, and thus observe a violation of Clauser, Horne, Shimony and Holt (CHSH) inequality by 2.7 standard deviations. The experimental setup is built using only fiber connections, which contribute to its long time stability.
\end{abstract}

Index Terms-Spontaneous four-wave mixing, Quantum entanglement, Sagnac fiber loop, Coincidence.

\section{INTRODUCTION}

Polarization-entangled photon pair sources are key technologies for realizing quantum communications [1]. In order to use them on real fiber networks, it's important that photon pairs can be generated in the $1550 \mathrm{~nm}$ wavelength band, where optical fibers have its minimum loss. The generation of polarization-entangled photon pairs using spontaneous fourwave mixing (SpFWM) in a Sagnac fiber loop, based on timemultiplexing, was presented in [2]. Later, Takesue and Inoue used the same principle, but the loop was based on polarization diversity [3]. Following that work, we implemented an experimental setup, using an highly nonlinear fiber (HNLF) instead of a dispersion-shifted fiber (DSF), to generate the entangledphoton pairs. Using a HNLF allows us to use lower pump powers, which decreases the noise detected by the avalanche photodiodes (APDs).

This paper contains five sections. In Section II we discuss local realism, including the Einstein, Podolsky, Rosen (EPR) argument, and Bell theory, that leads to the Clauser, Horne, Shimony and Holt (CHSH) inequality. In Section III, we present the experimental setup used to generate and detect polarization-entangled photon pairs. In Section IV, the experimental data are presented and discussed. The main conclusions of this paper are presented in Section $\mathrm{V}$

This work was partially supported by the Fundação para a Ciência e Tecnologia, FCT, through the Laboratório Associado (IT/LA) program, project "QuantTel - Quantum Secure Telecommunications" and "QuantPrivTel - Quantum Private Telecommunications" project (PTDC/EEATEL/103402/2008), FEDER and PTDC programs.

\section{LOCAL REALISM}

To perform the exchange of data using quantum cryptographic methods, one has to know and understand the basics, and all its baffling details. Two important concepts arise when one discusses the use of quantum cryptography for secure data transmission: the EPR argument and the Bell theory. The first made us aware of strange events related to physical phenomena, namely the interaction of particles at a given distance. The second is regarding a hypothetical explanation of these occurring events relying on the assumption of hidden local variables, which was lacked by the ruling theory.

\section{A. EPR Argument}

The EPR argument is considered by many as one of the major topics of quantum mechanics, mostly due to its importance in quantum cryptography. Einstein, Podolsky and Rosen questioned the reality of quantum mechanics and its truth in describing physical reality [4]. They stated that quantum mechanics wasn't correct, or at least it wasn't complete. Besides that, they argued that any theory had to describe in full all the phenomena which are covered by the theory. They expected that knowing the relation between two correlated quantities, one should know the values of both quantities and not be restricted to knowing one or the other, meaning that theoretical predictions have to agree with measurements performed on a given system. This can be mathematically stated by the Heisenberg uncertainty principle,

$$
\Delta r \Delta p \geq \frac{\hbar}{2}
$$

Eq. (1) regards the relative uncertainty between the position $(r)$ and the momentum $(p)$ of a given object, but can be applied to any pair of physically correlated quantities. Another disturbing detail is that the state of a given system is only defined when measured upon, with quantum mechanical theory claiming that the act of measuring inadvertently disturbs the system, disrupting the system's state. This was an unacceptable fact in their view. Another and very important phenomena that they observed was the action at a distance, where there is a dependency between two distant objects, known as "a spooky action at a distance". This action would be later on known as entanglement. 
In response to Einstein et al.'s paper, Niels Bohr answered with a paper of his own in which he states that the problem does not rely in the theory but in the way how measurements are performed, or more importantly, in the ways one analyzes a given phenomenon, focusing on the influence that experimental setups had over these measurements. With this, he introduced the concept of complementarity [5]. To further support his view, later were presented a series of examples based on the wave-particle duality of matter [6-8].

\section{B. Bell Theory}

Following the work of John von Neumann[9] and David Bohm [10, 11], John S. Bell devised a theory based on local hidden variables. In his paper, he presented a theorem based on nonlocality terms, while the locality theory is based on two assumptions [12]:

1) All objects have to be in a definite state from which the values of all other physical quantities can be determined, such as the position or momentum of an object.

2) The effects of local actions, such as measurements, cannot travel faster than the speed of light (as a result of special relativity). If the observers are sufficiently far apart, the measurement made by one will have no effect on the one done by the other (and vice-versa).

Considering the correlation between measurements, the causality condition imposed by local realism is upheld if the Bell theorem is proved correct. Item 11) addresses the issue of locality, while item (2) issues the separability criterion. The Bell theorem is stated as the following [12, 13],

$$
E(a, b)=\int_{\lambda \in \Lambda} p(\lambda) A(a, \lambda) B(b, \lambda) \mathrm{d} \lambda,
$$

where $\Lambda$ is the probability space, $\lambda$ are the hidden variables, with $A$ and $B$ being two physical quantities, while $a$ and $b$ are the axis at which $A$ and $B$ are projected over.

In order to test the quality of the entangled states, it can be measured the CHSH inequality, which is one type of the Bell inequalities. From (2), the polarization correlation coefficient, which requires four correlation measurements, can be defined as follows [14]:

$$
E\left(\theta_{1}, \theta_{2}\right)=\frac{C_{\theta_{1}, \theta_{2}}+C_{\theta_{1}^{\prime}, \theta_{2}^{\prime}}-C_{\theta_{1}, \theta_{2}^{\prime}}-C_{\theta_{1}^{\prime}, \theta_{2}}}{C_{\theta_{1}, \theta_{2}}+C_{\theta_{1}^{\prime}, \theta_{2}^{\prime}}+C_{\theta_{1}, \theta_{2}^{\prime}}+C_{\theta_{1}^{\prime}, \theta_{2}}} .
$$

In (3), $C_{\theta_{1}, \theta_{2}}$ are the coincidences between the polarizer of Alice set at angle $\theta_{1}$ and the polarizer of Bob set at angle $\theta_{2}$, being $\theta_{1}^{\prime}=\theta_{1}+90$ and $\theta_{2}^{\prime}=\theta_{2}+90$. In the CHSH inequality, the parameter $S$ is defined as [14]:

$$
S=\left|E\left(\theta_{1}, \theta_{2}\right)-E\left(\theta_{1}, \theta_{2}^{\prime}\right)+E\left(\theta_{1}^{\prime}, \theta_{2}\right)+E\left(\theta_{1}^{\prime}, \theta_{2}^{\prime}\right)\right|,
$$

and requires sixteen measurements. From quantum mechanics, the expectancy values for the CHSH inequality are,

$$
E\left(\theta_{1}, \theta_{2}\right)=E\left(\theta_{1}^{\prime}, \theta_{2}\right)=E\left(\theta_{1}^{\prime}, \theta_{2}^{\prime}\right)=\frac{1}{\sqrt{2}},
$$

and,

$$
E\left(\theta_{1}, \theta_{2}^{\prime}\right)=-\frac{1}{\sqrt{2}}
$$

These are the maximum values, and lead also to the maximum value of $S=2 \sqrt{2}$. This value can be obtained when polarization angles are set to $\left(\theta_{1}, \theta_{1}^{\prime}, \theta_{2}, \theta_{2}^{\prime}\right)=\left(0^{\circ}, 45^{\circ}, 22.5^{\circ}, 67.5^{\circ}\right)$. The uncertainty of the quantity $S$ is given by,

$$
\sigma_{S}=\sqrt{\sum_{i=1}^{16} C_{i}\left(\frac{\partial S}{\partial C_{i}}\right)^{2}},
$$

where the uncertainty of the $i$ th measurement, $C_{i}$, is $\sigma_{C_{i}}=\sqrt{C_{i}}$ [15].

To determine the existence of correlation between measurements, we estimate the values of each term in (4). If the sum is greater than 2, the Bell inequality is violated and this leads to physical reality being nonlocal, hence existing correlation between measurements. If not, local realism prevails and there is no correlation at all. Besides this, there are other limits and assumptions that need to be verified, regarding visibility and effective quantum efficiency values, among others. Considering quantum mechanics, there is also a maximum limit of correlation [16-18].

\section{EXPERIMENTAL SETUP}

In order to verify the effectiveness of our method of generation of polarization-entangled photon pairs, we used the setup presented in Fig. 1. A pump from a tunable laser source (TLS), with a full width at half maximum of $830 \mathrm{ps}$ and a repetition rate of $2.2 \mathrm{MHz}$ passes through an optical circulator, and a fiber Bragg grating (FBG), in order to eliminate the sidebands. The TLS is centered at $1550.918 \mathrm{~nm}$ wavelength, which is at the zero-dispersion wavelength of the HNLF used in the experiment. At the output of the optical circulator, the photons' polarization should be adjusted using a polarization controller (PC1), so that when they are focused in the Mach-Zehnder (MZ) modulator's $\mathrm{LiNbO}_{3}$ crystal has maximum efficiency. The MZ modulator is connected to a DC voltage source and a pattern generator. At the output of the $\mathrm{MZ}$ modulator is an erbium doped fiber amplifier (EDFA), which is used to amplify the pump's optical power. The noise from the EDFA is eliminated using a $100 \mathrm{GHz}$ flat fixed optical filter. In order to match the $45^{\circ}$ port of the polarization beam splitter (PBS), the

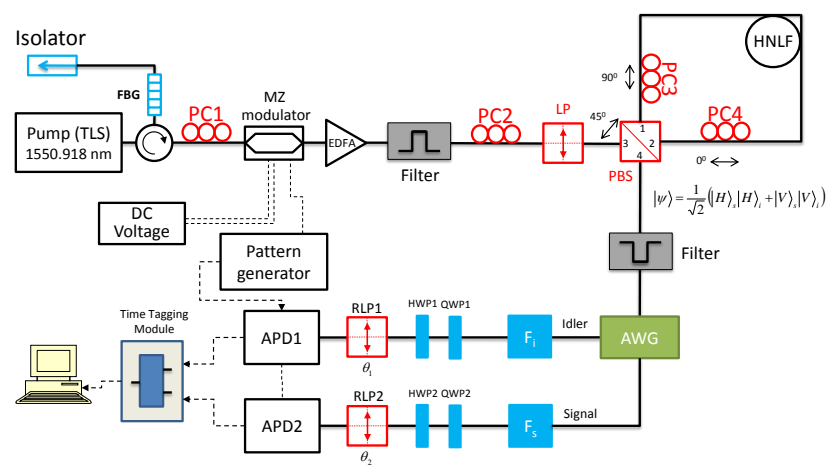

Fig. 1. Experimental scheme used for polarization-entangled photon pair generation through spontaneous four-wave mixing and coincidence detection. 

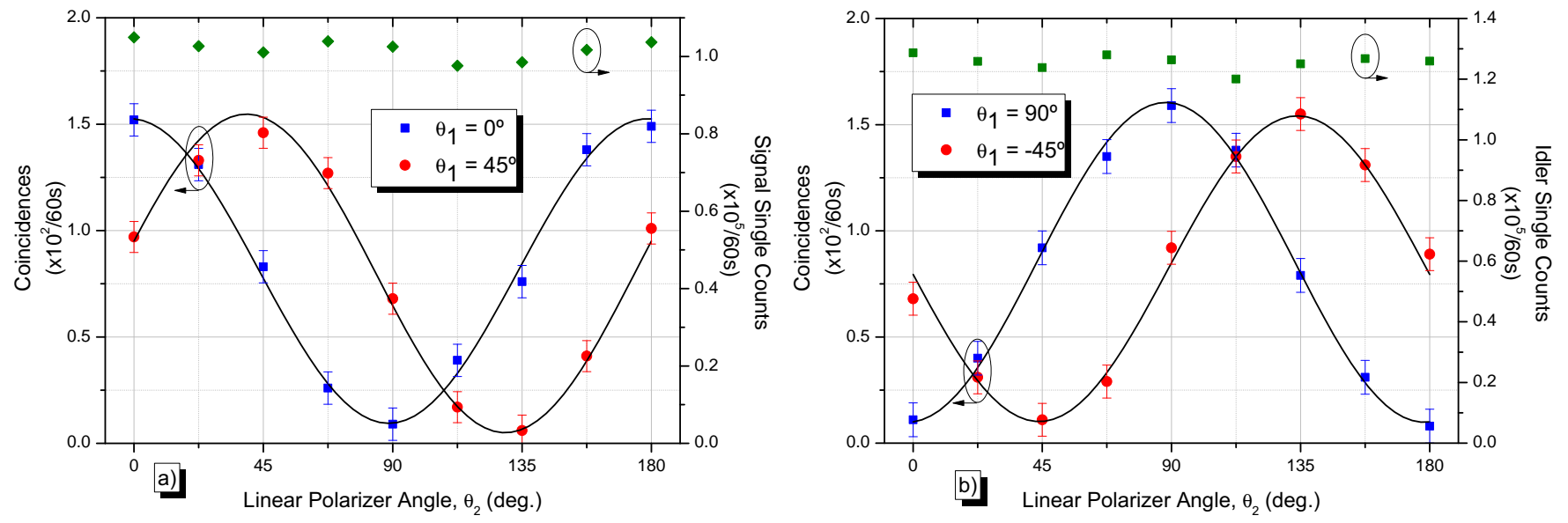

Fig. 2. Coincident and single counts as a function of $\theta_{2}$, while a) $\theta_{1}=0^{\circ}, 45^{\circ}$, and b) $\theta_{1}=90^{\circ}$, $-45^{\circ}$ were kept fixed. The solid curve is a sinusoidal fit to the experimental data. Error bars are a $5 \%$ deviation in relation to the maximum value, in each case.

photons are aligned with that port using another polarization controller (PC2) and a linear polarizer (LP) (note that the fiber between the LP and the PBS is polarization maintaining (PM) fiber). Then, the pulses are launched into the Sagnac loop, which consists in a 4-port PBS, a $150 \mathrm{~m}$ long HNLF with a nonlinear coefficient, $\gamma \sim 10.5 \mathrm{~W}^{-1} \mathrm{~km}^{-1}$, and the zerodispersion wavelength at $1550 \mathrm{~nm}$, and two PCs (PC3 and PC4). The power at the input of each arm of the HNLF was $2.2 \mathrm{~mW}$. Since the port- 3 of PBS is aligned at $45^{\circ}$ with the slow axis of the PM fiber, the PBS divides the pump pulses into horizontal $\left(0^{\circ}\right)$ and vertical $\left(90^{\circ}\right)$ polarization components, having each one equal optical power. The two components generate signal-idler photon pairs $|H\rangle_{s}|H\rangle_{i}$ and $|V\rangle_{s}|V\rangle_{i}$, while propagating in the loop in the counterclockwise and clockwise directions, respectively. The polarization controllers (PC3 and PC4) were adjusted so that the generated photon pairs were properly output from the loop, at port-4. The polarization-entangled photon pairs result in the superposition state given by,

$$
|\psi\rangle=\frac{1}{\sqrt{2}}\left(|H\rangle_{s}|H\rangle_{i}+|V\rangle_{s}|V\rangle_{i}\right)
$$

At the loop output the pump photons are eliminated, using another flat fixed optical filter [1]. Then, the idler and signal photons go into an arrayed waveguide grating (AWG), with a $100 \mathrm{GHz}$ channel spacing, which sends signal photons on one path, and idler photons to the other. AWG output channels with peak wavelengths of $\lambda_{s}=1547.715 \mathrm{~nm}$ and $\lambda_{i}=1554.134 \mathrm{~nm}$ were used for the signal and idler, respectively. Next, a cascade of flat fixed optical filters centered at the AWG's output wavelength channels are used in each arm in order to assure that only signal $\left(F_{s}\right)$ or idler $\left(F_{i}\right)$ photons pass, and all the other wavelengths are suppressed. The pump, signal and idler photons, with frequencies $\omega_{p}, \omega_{s}$ and $\omega_{i}$, respectively, satisfies the phase-matching condition, [3]:

$$
2 \omega_{p}=\omega_{s}+\omega_{i}
$$

The states of polarization of the signal and idler photons were adjusted using a quarter waveplate (QWP) and a half waveplate (HWP) so that the two photons experienced the same polarization change after they were separated by the AWG. Each photon was lead into a rotatable linear polarizer (RLP1 and RLP2) and was detected with an InGaAs/InP avalanche photodiode (APD1 and APD2) from IdQuantique, operating in a gated Geiger mode [19]. APD1 (id201) has a dark count probability per time gate, $t_{g}=2.5 \mathrm{~ns}$, of $P_{d c}<5 \times 10^{-6} \mathrm{~ns}^{-1}$, and a quantum detection efficiency, $\eta_{D} \sim 10 \%$ [20]. APD2 (id200) has a dark count probability per time gate, $t_{g}=2.5 \mathrm{~ns}$, of $P_{d c}<5 \times 10^{-5} \mathrm{~ns}^{-1}$, and a quantum detection efficiency, $\eta_{D} \sim 10 \%$ [21]. In order to avoid afterpulses, a $10 \mu$ s deadtime was applied to both detectors. The electric signals from the APDs were input into a time tagging module (TTM) for coincidence measurements. The TTM worked in a continuous mode, with a time resolution of 82.3 ps.

\section{EXPERIMENTAL RESUlTS}

In order to demonstrate polarization entanglement of the generated photon pairs experimentally, we inserted a polarization analyzer, before each APD, as shown in Fig. 1 . Each polarization analyzer consists of a QWP, a HWP and a RLP. We measured the coincidence rate for 16 combinations of polarizer settings $\left(\theta_{1}=-45^{\circ}, 0^{\circ}, 45^{\circ}, 90^{\circ} ; \theta_{2}=-22.5^{\circ}, 22.5^{\circ}\right.$, $67.5^{\circ}, 112.5^{\circ}$ ) in order to obtain the $S$ value of the CHSH inequality [22]. As stated before, any realistic theory must satisfy the condition $|S| \leq 2$. In order to verify the violation of CHSH inequality, it should be found that $|S|>2$.

In Fig. 2, we present coincidence and single counts detected over $60 \mathrm{~s}$, varying the RLP2 $\left(\theta_{2}\right)$, while the RLP1 $\left(\theta_{1}\right)$ is fixed for values $0^{\circ}, 45^{\circ}$ and $90^{\circ},-45^{\circ}$. Results for coincidence counts show that our source produces entangled photon pairs with strong correlation and a visibility greater than $86 \%$. In Fig. 22) we present signal single counts, as in Fig. 2p) are presented idler single counts, just as an example, since the 
other results obtained experimentally present similar behavior. The fact that single counts are roughly constant means that single photons are unpolarized or randomly polarized. This is a key characteristic of polarization-entangled photons [2].

In Table I we present the single and coincidence counts as a function of polarizer angles, $\theta_{1}$ and $\theta_{2}$ obtained experimentally.

TABLE I

SingLe $\left(N_{\theta_{1}}, N_{\theta_{2}}\right)$ AND COINCIDENCE $(C)$ COUNTS AS A FUNCTION OF POLARIZER ANGLES $\left(\theta_{1}, \theta_{2}\right)$.

\begin{tabular}{ccccc}
\hline \hline$\theta_{1}$ & $\theta_{2}$ & $N_{\theta_{1}}$ & $N_{\theta_{2}}$ & $C$ \\
\hline $0^{\circ}$ & $22.5^{\circ}$ & 102660 & 122880 & 131 \\
$90^{\circ}$ & $112.5^{\circ}$ & 102660 & 120060 & 138 \\
$0^{\circ}$ & $112.5^{\circ}$ & 97620 & 129000 & 39 \\
$90^{\circ}$ & $22.5^{\circ}$ & 105360 & 125880 & 40 \\
$0^{\circ}$ & $67.5^{\circ}$ & 103920 & 123420 & 26 \\
$90^{\circ}$ & $157.5^{\circ}$ & 98880 & 126780 & 31 \\
$0^{\circ}$ & $157.5^{\circ}$ & 101700 & 121500 & 138 \\
$90^{\circ}$ & $67.5^{\circ}$ & 97980 & 127980 & 135 \\
$45^{\circ}$ & $22.5^{\circ}$ & 102840 & 125280 & 133 \\
$-45^{\circ}$ & $112.5^{\circ}$ & 105840 & 119220 & 135 \\
$45^{\circ}$ & $112.5^{\circ}$ & 103260 & 123480 & 17 \\
$-45^{\circ}$ & $22.5^{\circ}$ & 102720 & 116040 & 31 \\
$45^{\circ}$ & $67.5^{\circ}$ & 101340 & 119940 & 127 \\
$-45^{\circ}$ & $157.5^{\circ}$ & 101880 & 124740 & 131 \\
$45^{\circ}$ & $157.5^{\circ}$ & 102660 & 117240 & 41 \\
$-45^{\circ}$ & $67.5^{\circ}$ & 99300 & 123780 & 29 \\
\hline
\end{tabular}

Using the experimental data we calculated the parameters defined in (3) and (4). The correlation coefficients required for the $\mathrm{CHSH}$ inequality and the respective errors are presented in Table II.

TABLE II

MEASURED CORRELATION COEFFICIENTS REQUIRED FOR THE CHSH INEQUALITY.

\begin{tabular}{ccccc}
\hline \hline$E\left(\theta_{1}, \theta_{2}\right)$ & $\left(0^{\circ}, 22.5^{\circ}\right)$ & $\left(0^{\circ}, 67.5^{\circ}\right)$ & $\left(45^{\circ}, 22.5^{\circ}\right)$ & $\left(45^{\circ}, 67.5^{\circ}\right)$ \\
\hline Value & 0.5460 & -0.6545 & 0.6962 & 0.5732 \\
Error & 0.0396 & 0.0445 & 0.0469 & 0.0418 \\
\hline
\end{tabular}

From the measured values we obtained a parameter $S=2.4699 \pm 0.1729$, when accidental coincidences were subtracted. Thus, we observed a violation of $\mathrm{CHSH}$ inequality by 2.7 standard deviations. This result is consistent with quantum mechanics.

\section{CONCLUSION}

We demonstrated the generation and detection of polarization-entangled photon pairs in the $1550 \mathrm{~nm}$ wavelength band, using SpFWM in a HNLF loop. When accidental coincidences were subtracted, we obtained a visibility for the coincidence fringes of more than $86 \%$, and thus observed the violation of $\mathrm{CHSH}$ inequality by 2.7 standard deviations.

\section{ACKNOWLEDGMENT}

The authors would like to thank Dr. Andreas Poppe from the Austrian Institute of Technology for lending the Time Tagging Module and for the helpful discussions, and Dr. Roland Lieger, also from the Austrian Institute of Technology, for the technical support regarding the Time Tagging Module.

We would also like to thank Prof. Paulo Monteiro and Nokia Siemens Networks S.A., Portugal, for lending the HNLF.

\section{REFERENCES}

[1] N. Gisin, G. Ribordy, W. Tittel, and H. Zbinden, "Quantum cryptography," Reviews of Modern Physics, vol. 74, pp. 145-195, Jan. 2002.

[2] X. Li, P. L. Voss, J. E. Sharping, and P. Kumar, "Optical-Fiber Source of Polarization-Entangled Photons in the $1550 \mathrm{~nm}$ Telecom Band," Physical Review Letters, vol. 94, no. 5, pp. 053 601-+, Feb. 2005.

[3] H. Takesue and K. Inoue, "Generation of polarization-entangled photon pairs and violation of Bell's inequality using spontaneous four-wave mixing in a fiber loop," Phys. Rev. A, vol. 70, no. 3, pp. $031802-+$, Sep. 2004.

[4] A. Einstein, B. Podolsky, and N. Rosen, "Can Quantum-Mechanical Description of Physical Reality Be Considered Complete?" Physical Review, vol. 47, pp. 777-780, May 1935.

[5] N. Bohr, "Can Quantum-Mechanical Description of Physical Reality be Considered Complete?" Physical Review, vol. 48, pp. 696-702, Oct. 1935.

[6] J. S. Bell, The measurement theory of Everett and de Broglie's pilot wave, Cambridge University Press, 2 ed., Ed., 2004.

[7] —_, de Broglie-Bohm, delayed-choice double slit experiment, and density matrix, Cambridge University Press, 2 ed., Ed., 2004.

[8] —, EPR correlations and EPW distributions, Cambridge University Press, 2 ed., Ed., Jun. 2004.

[9] J. von Neuman, Mathematische Grundlagen der Quantenmechanik, Springer, Berlin, Ed., 1932.

[10] D. Bohm, "A Suggested Interpretation of the Quantum Theory in Terms of "Hidden" Variables. I," Physical Review, vol. 85, pp. 166-179, Jan. 1952.

[11] _ "A Suggested Interpretation of the Quantum Theory in Terms of "Hidden" Variables. II," Physical Review, vol. 85, pp. 180-193, Jan. 1952.

[12] J. S. Bell, "On the Einstein-Podolsky-Rosen Paradox." Physics 1, pp. 165-200, 1964

[13] — "On the Problem of Hidden Variables in Quantum Mechanics," Reviews of Modern Physics, vol. 38, pp. 447-452, Jul. 1966.

[14] A. Aspect, P. Grangier, and G. Roger, "Experimental Realization of Einstein-Podolsky-Rosen-Bohm Gedankenexperiment: A New Violation of Bell's Inequalities," Physical Review Letters, vol. 49, pp. 91-94, Jul. 1982.

[15] D. Dehlinger and M. W. Mitchell, "Entangled photons, nonlocality, and Bell inequalities in the undergraduate laboratory," American Journal of Physics, vol. 70, pp. 903-910, Sep. 2002.

[16] B. d'Espagnat, "the quantum theory and reality," Scientific American, vol. 241, pp. 128-140, Nov. 1979

[17] J. S. Bell, The theory of local beables, Cambridge University Press, 2 ed., Ed., Jun. 2004.

[18] W. Y. Hwang, i. G. Koh, and Y. D. Han, "The detection loophole in Hardy's nonlocality theorem and minimum detection efficiency," Physics Letters A, vol. 212, pp. 309-314, Feb. 1996.

[19] G. Ribordy, N. Gisin, O. Guinnard, D. Stucki, M. Wegmuller, and H. Zbinden, "Photon counting at telecom wavelengths with commercial InGaAs/InP avalanche photodiodes: current performance," Journal of Modern Optics, vol. 51, pp. 1381-1398, Sep. 2004.

[20] idQuantique, id 201 Single-Photon Detector Module - Operating Guide, Version 4.0, 2008.

[21] — id 200 Single-Photon Detector Module - Operating Guide, Version $2.2,2005$

[22] J. F. Clauser, M. A. Horne, A. Shimony, and R. A. Holt, "Proposed Experiment to Test Local Hidden-Variable Theories," Physical Review Letters, vol. 23, pp. 880-884, Oct. 1969. 